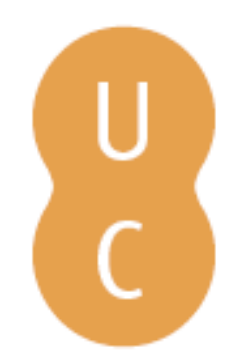

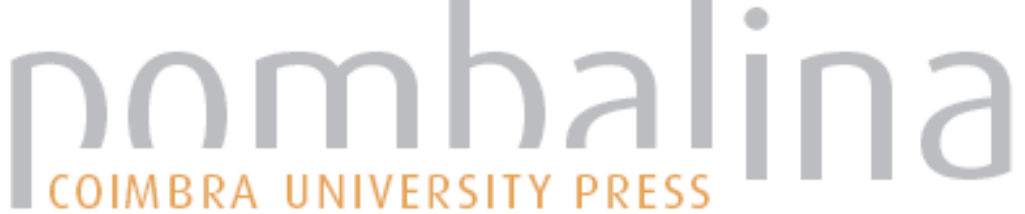

\section{Selbstbezüglichkeit und Geltung: ein methodenkritischer Beitrag zur Bewusstseinsphilosophie}

\author{
Autor(es): $\quad$ Grüneberg, Patrick \\ Publicado por: Imprensa da Universidade de Coimbra \\ URL \\ persistente: URI:http://hdl.handle.net/10316.2/31627 \\ DOI: $\quad$ DOI:http://dx.doi.org/10.14195/978-989-26-0205-9_8 \\ Accessed : $\quad$ 26-Apr-2023 11:37:50
}

A navegação consulta e descarregamento dos títulos inseridos nas Bibliotecas Digitais UC Digitalis, UC Pombalina e UC Impactum, pressupõem a aceitação plena e sem reservas dos Termos e Condições de Uso destas Bibliotecas Digitais, disponíveis em https://digitalis.uc.pt/pt-pt/termos.

Conforme exposto nos referidos Termos e Condições de Uso, o descarregamento de títulos de acesso restrito requer uma licença válida de autorização devendo o utilizador aceder ao(s) documento(s) a partir de um endereço de IP da instituição detentora da supramencionada licença.

Ao utilizador é apenas permitido o descarregamento para uso pessoal, pelo que o emprego do(s) título(s) descarregado(s) para outro fim, designadamente comercial, carece de autorização do respetivo autor ou editor da obra.

Na medida em que todas as obras da UC Digitalis se encontram protegidas pelo Código do Direito de Autor e Direitos Conexos e demais legislação aplicável, toda a cópia, parcial ou total, deste documento, nos casos em que é legalmente admitida, deverá conter ou fazer-se acompanhar por este aviso.

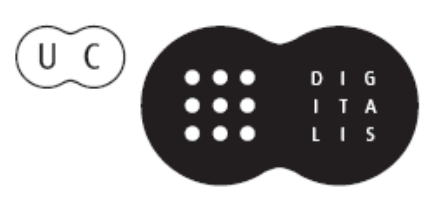


Edmundo Balsemão Pires

Burkhard Nonnenmacher

Stefan Büttner-von Stülpnagel

Editors

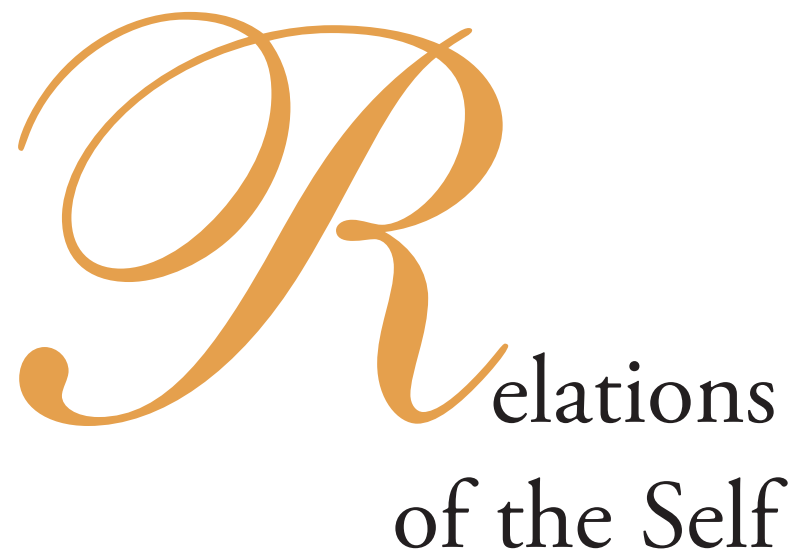




\section{Patrick Grüneberg \\ Berlin}

\section{SELBSTBEZÜGLICHKEIT UND GELTUNG - EIN METHODENKRITISCHER BEITRAG ZUR BEWUSSTSEINSPHILOSOPHIE}

Selbstbezüglichkeit ist ein in vielen Bereichen gebräuchlicher Begriff, um Systeme, Prozesse oder Strukturen zu bezeichnen, die sich auf sich selbst beziehen. Diesem Selbstbezug kommt dabei oft eine konstitutive Funktion zu. So führt der Selbstbezug des Subjekts zu Selbstbewusstsein, was für die Erkenntnistheorie von besonderem Interesse ist. Die reflexive Struktur des Bewusstseins fungiert im Rahmen der Transzendentalphilosophie und des Deutschen Idealismus als Bedingung sämtlicher Erkenntnis. Autopoietische Prozesse, mittels derer sich Lebewesen organisieren, werden in der Biologie ebenso als selbstbezüglich aufgefasst. In der formalen Logik und der Sprachphilosophie dienen selbstbezügliche Referenzen zur Klärung semantischer Antinomien. Im Folgenden steht der Zusammenhang zwischen Selbstbezüglichkeit und Geltung im Kontext philosophischer Bewusstseinstheorie in Zeutrum. Nun ist nicht unmittelbar klar, was unter einer explizit philosophischen Bewusstseinstheorie zu verstehen ist, da die Meinungen darüber, was Philosophie ist bzw. zu leisten imstande ist, weit auseinandergehen. Hier soll dafür argumentiert werden, dass es einen genuinen Bereich philosophischer Forschung gibt, nämlich den der Geltung. ${ }^{1}$ Eine philosophische Bewusstseinstheorie würde sich dann beispielsweise von einer psychologischen darin unterscheiden, dass letztere die Geltung bestimmter Grundbegriffe ungefragt voraussetzt. Die epistemische Reflexion auf die in Anschlag gebrachten Grundbegriffe würde dementgegen den damit einhergehenden Geltungsanspruch dieser Grundbegriffe allererst prüfen, bevor die eigentliche Bewusstseinstheorie formuliert würde. Letztlich hängt der Bereich der Geltung selbstverständlich mit den realwissenschaftlichen Erkenntnissen zusammen, so dass hier der

\footnotetext{
${ }^{1}$ Im naturalistischen Kontext, der hier einer grundbegrifflichen Analyse unterzogen wird, wird dieser Geltungsanspruch und damit die zentrale Aufgabe der Philosophie als Kohärenzanalyse der theoretischen Begriffe derjenigen Disziplinen verstanden, denen die Philosophie als begriffsklärendes und synoptisches Instrument beigegeben ist, allerdings ohne den dabei zugrundeliegenden Theorierahmen selbst in Frage zu stellen. Methodisch beruht dieser Philosophiebegriff auf der Antifundierungs-, Kontinuitäts- und Wissenschaftlichkeitsthese, die der Philosophie insgesamt keinen autonomen Erkenntnisanspruch zuerkennen; vgl. dazu Koppelberg, D.: „Was ist Naturalismus in der gegenwärtigen Philosophie?“, in: Keil, G. - Schnädelbach, H. (Hrsg.), Naturalismus. Philosophische Beiträge, Frankfurt a. M. 2000, S. 68-91, hier: S. 83 und Metzinger, T., Subjekt und Selbstmodell. Die Perspektivität phänomenalen Bewußtseins vor dem Hintergrund einer naturalistischen Theorie mentaler Repräsentation, Paderborn 1999, S. 26.
} 
Philosophie kein exklusiver Bereich zugesprochen wird. Es soll allerdings erwiesen werden, dass dieser Bereich der Geltung epistemologisch reflektiert werden muss, um eine gültige Bewusstseinstheorie zu formulieren. Indem die methodenkritische Analyse die jeweiligen Grundbegriffe auf ihre logisch-begriffliche Legitimation hin prüft, fungiert die Philosophie hier als kritischedisziplin, um theoretisch tragfähige Begriffe für eine konkrete Bewusstseinstheorie, welcher Provenienz auch immer, zu formmelieren. Aber auch im Rahmen philosophischer Bewusstseinstheorie verbleibt man nicht bei dieser propädeutischen Prüfung der Grundbegriffe. Diese müssen sich letztlich in der Formulierung der eigentlichen Bewusstseinstheorie bewähren und gegebenenfalls spezifiziert werden, wobei ein modifizierter Grundbegriff dann auch wieder hinsichtlich seiner Geltung geprüft würde, um seine explanatorische Rolle sicherzustellen. So versteht sich das methodenkritische Verfahren als eine kontinuierliche epistemologische Reflexion, die die jeweilige Bewusstseinstheorie ständig begleitet.

Zur Explikation des Zusammenhangs von Geltung und Selbstbezüglichkeit dienen zwei Thesen, deren eine ad absurdum geführt wird, um damit die andere zu begründen. Die zu widerlegende These beruht auf einem naturalistischen Ansatz, nämlich der Selbstmodelltheorie (SMT) Thomas Metzingers. Die These lautet: Das Bewusstsein als metamentale Repräsentation ist kausales Produkt des Gehirns. ${ }^{2}$ Damit ist zum einen der naturalistische Primat der Materie ausgesagt, d.h. die materielle bzw. biologische Wirklichkeit liegt dem Bewusstsein zugrunde, konstituiert selbiges. Da es sich beim Bewusstsein zum anderen um ein repräsentationales Gebilde handelt, lässt sich die Position der These als repräsentativer Realmonismus beschreiben: Die real existierende Natur bringt repräsentatives Bewusstsein hervor. Solche naturalistische Bewusstseinstheorie impliziert schließlich auch methodologisch einen Monismus, insofern die epistemologische Geltung dieser Art von Theorie auf einem erkenntnistheoretischen Realismus und somit auf der veranschlagten Realität der Natur beruht. Versteht man demgegenüber unter einer Wissenstheorie die oben angedeutete grundbegriffliche Analyse und insbesondere den damit implizierten Bereich philosophischer Geltung, dann lässt sich der repräsentative Realmonismus unter dem Motto „Bewusstseinstheorie ohne Wissenstheorie“ zusammenfassen, weil in epistemologischer Hinsicht kein eigenständiger Bereich logischbegrifflicher Geltung angenommen wird. Selbstbezüglichkeit besteht in SMT nur auf der Ebene der neuronalen Instantiierung von Repräsentationen, insofern sich diese durch Metarepräsentationen auch wieder selbst abbilden bzw. modellieren.

Die Gegenthese umfasst hier mit Blick auf die reductio ad absurdum die Negation der These: Das Bewusstsein ist nicht monokausales bzw. ausschließliches Produkt der Natur und damit irreduzibel selbstbezüglich. Es ist keine vollständige naturalistische Objektivation des Bewusstseins möglich, weil auch schon eine solche Objektivation die Leistungen bzw. Vermögen des in Frage stehenden Objekts, hier des Bewusstseins, in Anspruch nimmt. Diese Leistungen geben keinen objektiven Blick auf den Gegenstand, d.h. die wissenslogischen Voraussetzungen steuern bereits auch schon die Konstituierung dessen, was wir unter dem realistischen Begriff der biologischen Wirklichkeit fassen. Kurz gesagt: „Keine Bewusstseinstheorie ohne Wissenstheorie“, d.h. die Reflexion auf die grundbegrifflichen Voraussetzungen ist notwendig, um gültige Aussagen über das Bewusstsein treffen zu können. Im Gegensatz

\footnotetext{
${ }^{2}$ Anstelle des Gehirns wird auch das gesamte zentrale Nervensystem als Produzent angeführt.
} 
zum Realmonismus handelt es sich bei dieser kritischen Position um einen transzendentalen Dualismus, der die Form des Bewusstseins eigens reflektiert, um damit die Voraussetzungen zu klären, unter denen das Bewusstsein überhaupt thematisiert werden kann. Der wissenstheoretische Anteil umfasst diese transzendentale Reflexion als eine reflexive Epistemologie mit einem eigenen, d.h. hier insbesondere nicht-empirischen Geltungsanspruch: Wissen basiert nicht ausschließlich auf der biologischen Wirklichkeit, sondern beruht auch auf einer gegenüber der Natur eigenständigen epistemischen „Sphäre“. Diese strukturell selbstbezügliche Sphäre wird in der folgenden Untersuchung herausgearbeitet und in ihrer Irreduzibilität erwiesen. Es wird sich zeigen, dass eine gültige Bewusstseinstheorie unter Berücksichtigung der Selbstbezüglichkeit des Bewusstseins möglich ist.

\section{Zur Vorgehensweise}

Ziel der folgenden Untersuchung ist der Erweis der Ungültigkeit der These. Die Kritik an SMT kann dabei grundsätzlich zwei Formen annehmen. Zum einen könnte faktisch gezeigt werden, dass SMT bzw. der naturalistische Repräsentationalismus nicht erklären kann, wie unser reflexiver Bezug zur empirisch gegebenen Realität möglich ist, d.h. nicht erklären kann, wie wir einen objektivierenden Begriff unserer selbst als natürlicher Wesen in einer vorfindlichen Natur bilden, diesen wissenschaftlich instrumentalisieren können und wie wir aufgrund dieses reflexiven Verhältnisses zu uns und der gegebenen Wirklichkeit zweckorientiert handeln können. Hier ist allerdings keine faktische Argumentation beabsichtigt, d.h. keine Kritik an naturalistischen Begriffen, die darauf basiert, deren Geltungsanspruch durch den Verweis auf die fehlende Erklärungskraft, die zur Klärung der genannten faktischen Bewusstseinshandlungen notwendig sind, einzuschränken. Stattdessen soll in logischbegrifflicher Hinsicht eine Beweisführung erbracht werden: Wenn gezeigt werden kann, dass SMT bzw. der repräsentative Realmonismus prinzipiell an der Erklärung von Bewusstsein scheitern müssen, ${ }^{3}$ kann im Umkehrschluss aus dem Beweis der Unmöglichkeit des Gegenteils geschlossen werden, dass die Gegenthese gültig ist, nämlich dass das Bewusstsein und unser gesamtes Selbst- und Weltverhältnis genuin selbstbezüglich und damit naturalistisch irreduzibel sind. Das ist die Form des apagogischen (indirekten) Beweises bzw. der reductio ad absurdum. Die zu beweisende Aussage („Keine Bewusstseinstheorie ohne Wissenstheorie“ bzw. der transzendentale Dualismus) wäre also durch den Nachweis der Unmöglichkeit ihres Gegenteils, d.h. dass realmonistische Grundbegriffe in Aporien bzw. zu Widersprüchen führen, erwiesen. Daraus resultiert folgende Gliederung:

(1) Darstellung der These (SMT) anhand einer grundbegrifflichen Analyse: Bewusstsein ist Repräsentation bzw. das Produkt neuronaler Modellierung.

(2) Vorbemerkungen zum Verfahren der methodenkritischen Analyse

${ }^{3}$ Die hier zugrundegelegten Prinzipien sind der Satz des zureichenden Grundes, der Satz des Widerspruches sowie die Identität bei Nichtunterschiedenheit, gehören also dem Bereich der Logik an und konstituieren in begriffslogischer Hinsicht die Möglichkeit, sinnvoll argumentieren zu können. 
(3) Kritische Prüfung der Grundbegriffe: Die naturalistischen Grundbegriffe fallen dem Münchhausen-Trilemma anheim.

(4) Beweis der Gegenthese: Bewusstseinstheorie ohne Wissenstheorie ist ungültig.

(5) Das geltungstheoretische Problembewusstsein und die formale Selbstbezüglichkeit des Bewusstseins: Zusammenfassung und Ausblick

\section{SMT: grundbegriffliche Analyse}

Der Begriff der Repräsentation kommt seit jeher in den unterschiedlichsten Feldern der Philosophie zur Anwendung, man kann ihn durchaus zurecht als einen philosophischen Grundbegriff bezeichnen, der trotz der starken Diversifizierung wissenschaftlicher Philosophie eine durchgehende Prominenz behauptet.

\section{Zum Zusammenhang von Repräsentation und Bewusstsein in der Philosophie des Geistes}

SMT widmet sich zentral der Frage, wie unbewusste Ereignisse und Zustände durch eine repräsentationale Erfassung mental und somit bewusst werden können. Diese Frage resultiert aus dem Zusammenhang von Repräsentation und Bewusstsein, der in der Philosophie des Geistes nach Esken und Heckmann dahin gehend problematisiert wird, ob und inwiefern Bewusstsein einen univoken Sinn hat, d.h. für systemische und subsystemische Zustände, Ereignisse und Prozesse gleichermaßen gilt.

Zur Klärung dieser Frage dient die Unterscheidung zwischen Bewusstsein als ursprüngliche und als metamentale Repräsentation. Bewusstsein lässt sich im Sinne eines Merkmals zum einen als „Globalcharakteristikum eines wachen Handlungssystems“ ${ }^{\text {« }}$ betrachten. Darunter fallen sensorische, kognitive, volitive sowie behaviorale Fähigkeiten, die nicht nur dispositional (im Sinne möglicher Handlungsweisen des Systems), sondern auch episodisch manifestiert sind. Die Wahrnehmung von Dingen und Ereignissen schließt wiederum die mentale Verarbeitung dieser Wahrnehmungen und auf Dinge bezogenen Gedanken selbst mit ein. Zum anderen lässt sich Bewusstsein aber auch „als Merkmal von subsystemischen Ereignissen, Prozessen und Zuständen"s thematisieren. Als paradigmatischer Fall wird in diesem Zusammenhang gerne der Traum angeführt: Dieser stellt eine mentale Episode dar, die für den Träumenden während des Traums bewusst ist, aber andererseits nicht auf den Träumenden als Gesamtsystem zutrifft. Bewusstsein in diesem Sinne umfasst dann jeweils einzelne sensorische Zustände und Ereignisse, d.h. die verschiedenartigen Wahrnehmungen, propriozeptive Empfindungen, bildhafte Vorstellungen sowie bestimmte kognitive und volitive Zustände im Sinne manifester propositionaler Einstellungen, also propositionale Akte des Denkens, Urteilens, Meinens, Verstehens, Entscheidens, Beabsichtigens und Wollens

\footnotetext{
${ }^{4}$ Esken, F. - Heckmann, D., „Generelle Einführung: Bewußtsein und Repräsentation. Bemerkungen über zwei Schlüsselbegriffe, ihre Ausdifferenzierung und ihren Zusammenhang“, in: Esken, F. - Heckmann, D. (Hrsg.), Bewußtsein und Repräsentation, Paderborn 1998, S. 11-49, hier: S. 15.

${ }^{5}$ Esken/Heckmann 1998, S. 15.
} 
sowie Prozesse des Deliberierens und Konkludierens und Emotionen, Gefühle, Stimmungen und Affekte. ${ }^{6}$

Bei so unterschiedlichen Phänomenen, die alle als Bewusstsein gelten, stellt sich natürlich die Frage nach dem gemeinsamen Merkmal dieser als bewusst bezeichneten Zustände und Vorgänge. Der gemeinsame Nenner ist die gängige Bestimmung von Bewusstsein als Erleben. Anknüpfend an das bekannte Theorem des „What is it like to be“ von T. Nagel" ergeben sich hier drei Merkmale für das bewusste Erleben, die zugleich Subjektivität fundieren sollen:

(a) Erlebnisse sind immer subjektiv, d.h. immer Erlebnisse für ein System;

(b) Erlebnisse haben einen phänomenalen bzw. qualitativen Gehalt, ein Qualia;

(c) Erlebnisse erlauben nicht die Unterscheidung zwischen sein und zu sein scheinen, d.h. die Erlebnisqualität hängt nicht davon ab, wie das Subjekt diese Erlebnisse bewertet.

Allerdings wirft diese Bestimmung von Bewusstsein als Erleben wiederum die Ausgangsfrage auf, da das Merkmal des Erlebens nur schwerlich auch für nicht-phänomenale Akte und schon gar nicht für subsystemische Zustände gelten kann. Besitzen etwa Akte des Meinens oder Fürwahrhaltens einen phänomenalen Gehalt? Generell ergibt sich das Problem, dass „[f]ür Perzeptionen und Propriozeptionen phänomenaler Gehalt und subjektive Erlebnisperspektive essentielle Merkmale [sind], während sie für propositionale Akte bestenfalls akzidentellen Charakter haben. " 8 Die hieraus für eine Bestimmung des Bewusstseinsbegriffes entstehende Disparatheit stellt sich als eine Alternative dar: Entweder der hier bestimmte Bewusstseinsbegriff umfasst nur offenkundig phänomenale Akte, wie sensorische bzw. sinnlich-phänomenale Zustände, allerdings mit der Konsequenz, dass bewusste propositionale Akte dann nach einer anderen Bestimmung verlangen. Oder das phänomenalistische Kriterium des Erlebtwerdens muss verworfen werden, um doch noch einen univoken Sinn für phänomenale wie nicht-phänomenale und vor allem subsystemische Zustände formulieren zu können.

Zur Lösung dieser Schwierigkeit tritt die Repräsentation in zwei konkurrierenden Gestalten auf den Plan, nämlich als ursprüngliche und als metamentale Repräsentation. Beiden Varianten liegt das Theorem der Intentionalität des Bewusstseins zugrunde, nämlich dass Bewusstsein immer Bewusstsein von etwas ist. Der Repräsentationsgehalt umfasst in einem solchen intentionalen Verhältnis die sinnlich wahrnehmbaren Dinge und Ereignisse, während das Repräsentationsvehikel für die Bewusstwerdung dieses Gehaltes verantwortlich ist. Im Rahmen der Konzeption des Bewusstseins als ursprüngliche Repräsentation wird die zuvor festgestellte Disparatheit von phänomenalen Erlebnisinhalten und propositionalen Akten hinsichtlich des subjektiven Erlebnischarakters eingeholt, indem zwischen sensorischen und propositionalen Repräsentationen unterschieden wird. Durch diese Subsumierung aller Bewusstseinszustände unter den Begriff der Repräsentation fungieren alle subsystemischen Zustände und Ereignisse als Repräsentationen, weil sie dem Gesamtsystem etwas bewusst machen. Sensorische Repräsentationen gehen dabei auf sinnliche Erfahrung und die derart vermittelten phänomenalen Qualitäten, während propositionale Repräsentationen auf

\footnotetext{
${ }^{6} \mathrm{Vgl}$. ebd.

${ }^{7}$ Nagel, Th., „What is it like to be a bat?”, in: Philosophical Review 83 (1974), S. 435-450.

${ }^{8}$ Esken/Heckmann 1998, S. 19.
} 
propositionale Akte referieren. Damit gilt: „Bewußtsein ist ursprüngliche Repräsentation [,] und

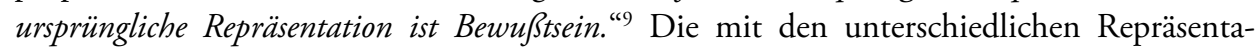
tionsgehalten (sinnlich-phänomenal bzw. propositional) auftretende notwendige Unterscheidung wird somit der internen Struktur des Repräsentationssystems übertragen, das dafür verschiedene Klassen von Repräsentationsvehikeln bereitstellen muss.

Die metamentale Repräsentation kehrt das Verhältnis zwischen den Ereignissen bzw. Zuständen und dem System um: Die gegebenen Ereignisse und Zustände sind dem System nicht deshalb bewusst, weil sie als ursprünglich immer schon repräsentational nur noch erlebt zu werden brauchen, sondern sie sind bewusst, weil das System sie durch repräsentationale Prozesse allererst repräsentational erfasst und damit bewusst macht. Diese repräsentationale Erfassung selbst ist allerdings unbewusst, während die Bewusstwerdung, also das phänomenale Erleben, durch eine weiteremetarepräsentationale Erfassung erfolgt, die ihrerseits auch unbewusst ist. Der entscheidende kritische Punkt an der Metarepräsentation liegt dabei in der Frage, wie unbewusste Ereignisse und Zustände durch eine metarepräsentationale Erfassung mental und somit bewusst werden können.

\section{Bewusstsein durch Metareprësentation: die Selbstmodelltheorie}

SMT geht auf den modelltheoretischen Ansatz K. Craiks zurück, der mit der Hypothese, dass „das Gehirn mit analogen internen Modellen von Aspekten der Außenwelt [operiere] “10, maßgebende Vorarbeiten geliefert hat. Zur Beantwortung der entscheidenden Frage nach den Konstitutionsbedingungen für phänomenales Bewusstsein wird mit SMT eine Repräsentationsarchitektur geschaffen, innerhalb derer Bewusstsein bzw. Selbst- und Weltbezug durch eine metarepräsentationale Modellierung bereits vorliegender Repräsentate der Außenwelt und des repräsentierenden Wesens selbst erzeugt werden. Mentale Repräsentation stellt damit einen Vorgang dar, durch den der Kognition fähige Lebewesen bzw. Biosysteme innere Beschreibungen bzw. Abbildungen von einzelnen Bereichen der Wirklichkeit erzeugen. Für uns Menschen als ein solches Biosystem folgt daraus, dass „[d]ie Inhalte unseres Bewußtseins die Resultate einer von unseren Gehirnen erbrachten Repräsentationsleistung [sind], deren Mechanismus wir derzeit immer besser zu verstehen beginnen. ${ }^{\text {"11 }}$ Dabei rückt die Mentalität dieser Repräsentationen als der (zumindest für uns Menschen entscheidende) „Sonderfall biologischer Informationsverarbeitung ${ }^{\text {"12 }}$ in den Fokus der Untersuchung. Die als Zustände bestimmten Repräsentationen, die wir als mental bzw. als bewusst erleben, sind ihrerseits nämlich nur eine bestimmte Klasse von informationsverarbeitenden Zuständen überhaupt. Interne Systemzustände wie beispielsweise biologische Informationsverarbeitung (Steuerung des Herzschlages oder die Aktivität des Immunsystems) werden gerade dann mental, wenn

\footnotetext{
${ }^{9}$ Ebd., S. 28.

${ }^{10}$ Scholz, O.R., „Repräsentation, III. 19. und 20. Jh.“, in: Ritter, J. - Gründer, K. (Hrsg.), Historisches Wörterbuch der Philosophie, Darmstadt 1971, Band 8, Sp. 827-834, hier: Sp. 832. Siehe auch Craik, K., The nature of explanation, Cambridge 1943 sowie Johnson-Laird, P. N., Mental models, Cambridge 1983.

${ }^{11}$ Metzinger 1999, S. 47.

${ }^{12}$ Ebd., S. 47f.
} 
sie Inhalt einer höherstufigen Repräsentation werden - und das gilt nicht für alle Zustände gleichermaßen. SMT widmet sich daher der Frage, wie interne Repräsentationen zu mentalen Repräsentationen werden, d.h. „zu Prozessen, die potentielle Inhalte subjektiven Bewußtseins sind." ${ }^{33}$

Mittels des Begriffs der Repräsentation ist Bewusstsein also grundlegend als eine Repräsentationsbeziehung zwischen einer gegebenen Wirklichkeit und dem repräsentierenden kognitiven System bestimmt, die insofern mental ist, als dass die Repräsentation selbst als geistige Entität verstanden wird. Diese Beziehung wird in SMT als eine systeminterne analoge Modellverarbeitung konzipiert. Die formale Struktur der mentalen Repräsentation ist folgendermaßen als dreistellige Relation bestimmt: $M_{\text {Rep }}(S, X, Y)$, will heißen: Der interne Zustand X repräsentiert einen Bereich der Welt Y für das System S. Repräsentation ist somit „ein Prozeß, der für ein System die innere Beschreibung eines Repräsentandums [das ist der Repräsentationsgehalt; P.G.] durch die Erzeugung eines als Repräsentat [das ist das Repräsentationsvehikel; P.G.] fungierenden Zustands leistet. "14 ${ }^{14}$ as Repräsentandum bilden (in Bezug auf das System S) externe und interne Faktoren, Ereignisse oder Prozesse bzw. die „Sachverhalte der Welt" ${ }^{15}=$ Y. Mit der internen Repräsentation von Aspekten der Außenwelt wird ein Realitätsmodell konstituiert.

Weil die realisierten Datenstrukturen (X) selbst nicht phänomenal erlebt werden bzw. keine ursprünglichen Repräsentationen darstellen, bedarf es einer höherstufigen Repräsentation, d.h. einer metamentalen Repräsentation, um eine andere Repräsentation bewusst zu machen. ${ }^{16}$ Ausgehend von der repräsentationalen Modellierung der biologischen Wirklichkeit bzw. dem Realitätsmodell, das als solches noch nicht zu Bewusstsein führt, erzeugt das Lebewesen weiterhin ein Selbstrepräsentat. Dieses Selbstmodell wird durch das System „in das von ihm aktivierte Realitätsmodell ein[ge]bettet ${ }^{\text {"17 }}$, so dass Subjektivität ${ }^{18}$ relational aus dem Zusammenhang von Realitäts- und Selbstmodell instantiiert wird. Selbstbewusstsein bzw. bewusstes subjektives Erleben seiner selbst resultiert schließlich aus solchen „Partitionen des Selbstmodells, die prinzipiell durch Metarepräsentationen zu Inhalten phänomenalen Bewusstseins werden können. ${ }^{\text {"19 }}$ Das phänomenale Selbst bzw. das Subjekt innerer Erlebnisse ist dann „[d]erjenige Teil des mentalen Selbstmodells, der durch Metarepräsentation zum aktuellen Gehalt phänomenalen Bewußtseins wird." ${ }^{20}$ Subjektivität insgesamt wird somit bestimmt als eine spezifische Eigenschaft informationsverarbeitender Systeme, die ein Selbstmodell in ihr Realitätsmodell einbetten, also „Selbstmodellbesitzer“21 sind.

${ }^{13}$ Ebd., S. 48.

${ }^{14}$ Ebd., S. 51.

${ }^{15}$ Ebd., S. 52.

${ }^{16}$ Mit Blick auf mensdiliches Bewnsstsein, beziche ich mich im Folgenden auch auf selbstbewusstsein, d, h. Bewusstsein im ganzer als ein welt- und selbsbezug. In seiner repräsentationalen genese des Bewusstsein, hält Metzinger auch nicht-zentriertes Bewusstsein, also die metarepräsentationale Erfassung von Elementen des Realitätsmodells ohne ein eingebettetes Selbstmodell für möglich; vgl. dazu die Bemerkungen zum aperspeletivistischen Bewusstsein ohne Subjektivität, ebd. S. 264.

${ }^{17}$ Ebd., S. 204.

${ }^{18}$ bzn. ein selbstbezug und damit Metzinger zufolge eine Zentrierung des Bewusstsein.

${ }^{19}$ Ebd., S. 159.

${ }^{20}$ Ebd.

${ }^{21}$ Ebd., S. 205. 
Neben der Repräsentation werde ich im Folgenden zwei weitere tragende Grundbegriffe naturalistischer Theoriebildung zur methodenkritischen Prüfung heranziehen. Das repräsentationale Welt- und letztlich auch das Selbstverhältnis beruhen in wirklichkeitskonstitutiver Hinsicht gemäß des Realmonismus auf einem erkenntnistheoretischen bzw. repräsentativen Realismus. Mit Blick auf die biologische Entstehung lassen sich Selbstmodelle, die nach dem heutigen Stand der Forschung nur Biosystemen zukommen, als „abstrakte Organe, die von solchen Systemen ausgebildet wurden und ihnen anscheinend einen Auslesevorteil ermöglicht haben" ${ }^{22}$, beschreiben, womit dem Teleofunktionalismus in handlungskonstitutiver Hinsicht eine tragende Funktion zukommt.

\section{Realismus}

Generell (also organismusunabhängig) gilt mit Blick auf die Funktionalität des Selbstmodells, dass „[z]um repräsentationalen Gehalt von Selbstmodellen eine bestimmte Teilmenge der durch das System erfaßbaren kausalen Eigenschaften der Welt [gehört], nämlich genau diejenigen kausalen Eigenschaften, die vom System sehr leicht, direkt und zuverlässig verändert werden können. "23 SMT setzt diese Welt sowie deren kausale Eigenschaften inklusive deren Zugänglichkeit voraus und ist somit in epistemologischer Perspektive als realistisch zu beurteilen, d.h. dass ein ontologisch unabhängiges und zugleich epistemisch zugängliches Sein vorausgesetzt wird, wobei dieses Sein gemeinhin mit der Natur in eimen biologischen sinne identifiziert wird. Als Explanans des zu erklärenden phänomenalen Bewusstseins fungiert der biologisch-organische Körper des Organismus bzw. des Systems als dem Träger dieses Bewusstseins einschließlich der Umwelt. Die epistemische Zugänglichkeit liegt bereits in dem grundlegenden Repräsentationsverhältnis inbegriffen, mit dem gesetzt wird, dass eine repräsentationale Erfassung der gegebenen Wirklichkeit generell möglich ist. Als „innere Werkzeuge der Informationsverarbeitung “24 erfüllen Selbstmodelle so die Aufgabe, „die relationale Struktur ihrer Gegenstände so vollständig wie möglich darzustellen. “25 Damit wird die „Notwendigkeit eines grundsätzlichen ,In-der-Welt-Seins'“ vorausgesetzt d.h. „daß es subjektive Inhalte des Bewußtseins nur dann geben kann, wenn es auch objektive Inhalte gibt." ${ }^{26}$

\section{Teleofunktionalismus}

Neben der Bestimmung des konstitutiven Realitätsbezuges bildet die Konzeption der praktischen Handlungsfähigkeit des kognitiven Systems einen weiteren entscheidenden Bestandteil jeglicher Bewusstseinstheorie. Auch Handlungen werden durch Selbstmodelle

\footnotetext{
22 Ebd., S.159.

${ }^{23}$ Ebd., S. 160, Anm. 15.

${ }^{24}$ Ebd., S. 169.

${ }^{25}$ Ebd.

${ }^{26}$ Ebd., S. 207.
} 
konstituiert, indem diese als Werkzeuge fungieren. Der Werkzeugcharakter des Selbstmodells beruht dabei auf einer evolutionären Ausrichtung. Mit Blick auf die „biologische Funktion“ als „Instrumente oder Waffen“27 erfüllt ein Selbstmodell einen praktischen Zweck: es lässt das System zu einem Agenten werden. Im Gegensatz zu bis dato bekannten künstlichen Systemen, die keine (bzw. nur vom Programmierer vorgegebene) Interessen verfolgen, ist ein organisches System erst dann handlungsfähig, wenn mittels des teleologischen Zusatzkriteriums benannt werden kann, in welcher Hinsicht die realistisch-repräsentationale Erfassung der Wirklichkeit relevant ist, d.h. welche kausalen Eigenschaften das System für sich nutzen kann - und relevant ist dem Evolutionismus zufolge die Selbst- und Arterhaltung und damit die Auswahl entsprechender kausaler Zusammenhänge. Die repräsentationale Modellierung systemspezifischer Eigenschaften in Bezug auf kausale Verhältnisse in der Welt kann also nur mittels eines teleologischen Zusatzkriteriums zu einem handlungsfähigen System führen. Dem System werden auf diese Weise Absichten beigelegt, nach denen es handelt, ohne dass dafür ein handelndes Subjekt veranschlagt wird. Der Agent ist das System als Ganzes. ${ }^{28}$ Gemäß des Teleofunktionalismus bezieht sich also jegliche Funktionalität der Selbstmodelle ausschließlich auf eine „optimale Verhaltenssteuerung relativ zu einer gegebenen Umwelt “29, weil das menschliche Selbstmodell aus einem „'kognitiven Wettrüsten’ auf unserem Planeten [...], das relativ zu der spezifischen biologischen Umwelt des Menschen auf diesem Planeten zu einer Optimierung von Funktionalität führte “30, entstanden ist. So wird das menschliche Bewusstsein hinsichtlich seiner Praktizität bzw. seines Handlungsvermögens mittels des Evolutionismus in den übergeordneten Zusammenhang der biologischen Evolution eingeordnet und insgesamt naturalisiert. ${ }^{31}$

\section{Vorbemerkungen zur methodenkritischen Analyse}

Es stellt sich mit Blick auf die epistemologische Geltungsfrage die kritische Notwendigkeit, das Vorgehen der Prüfung selbst zu klären, d.h. anzugeben, welche Geltungskriterien der Prüfung zugrunde liegen bzw. wie die Widersprüche, die zur reductio ad absurdum des Realmonismus führen, expliziert werden. Was für die empirischen Wissenschaften konstitutiv ist, muss dies nicht auch in gleichem Maße für einen epistemologisch basierten Diskurs sein, der vermeintliche Selbstverständlichkeiten methodisch hinterfragt. Während man sich im wissenschaftlichen Betrieb gemeinhin der jeweiligen disziplinären Grundbegriffe und Methoden bedient, stehen im Rahmen einer Methodenkritik die entsprechenden Grundbegriffe der Methoden bzw. Verfahrensweisen selbst auf dem Prüfstand. Mittels dieser Grundbegriffe können Hypothesen gebildet und somit Erklärungen für bestimmte

${ }^{27}$ Ebd.

${ }^{28}$ Vgl. dazu Metzinger 1999, S. 55 und S. $152 \mathrm{ff}$.

${ }^{29}$ Ebd., S. 170.

${ }^{30}$ Ebd., S. 170.

${ }^{31}$ Vgl. zum Begriff des Teleofunktionalismus Sober, E.: „Putting the Function back into Functionalism“, in: Lycan, W.G. (Hrsg.), Mind and Cognition. A Reader, Cambridge (MA) und Oxford 1990, S. 97-106. 
Phänomene formuliert werden. Die Frage nach der Geltung zielt auf die logisch-begriffliche Gültigkeit einer Theorie und damit auf diese Grundbegriffe ab. Der Bestand an Grundbegriffen, den eine Theorie umfasst, ist dabei aus zweierlei Gründen interessant: Das offensichtliche Interesse - auch für den jeweiligen Wissenschaftler - liegt im Erklärungswert der Grundbegriffe, also den Möglichkeiten, die diese bieten, die Struktur und Funktionalität bestimmter Phänomene bestimmen zu können (Erklärungsebene). Weniger offensichtlich ist die Voraussetzungsebene einer Theorie, die in jeder Erklärung insofern immer schon mitgegeben wird, als dass die epistemische Gültigkeit der verwendeten Grundbegriffe in Anspruch genommen wird, sobald eine Erklärung, aber auch schon eine Hypothese formuliert werden. Die Methode einer Theorie wird hier also anhand der Grundbegriffe kritisch untersucht.

\section{Das methodenkritische Analyseschema im Überblick}

Als Ausgangspunkt der Analyse dient die Unterscheidung zwischen der Voraussetzungsund der Erklärungsebene der in Frage stehenden Theorie. Seine Gültigkeit verdankt ein Grundbegriff dabei seinem terminus a quo bzw. einem Gefüge mehrerer Begriffe, die zusammen den terminus a quo bilden. Die Erklärungsebene umfasst das Erklärungsziel bzw. den terminus ad quem (Abb. 1). ${ }^{32}$

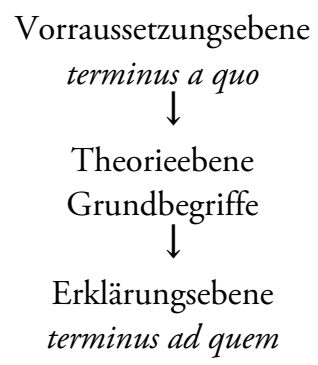

(Abb. 1)

Die epistemische Gültigkeit eines Grundbegriffs wird gewährleistet durch den terminus a $q u o$, indem dieser in Form logisch-begrifflicher Bedingungen der Möglichkeit des jeweiligen Grundbegriffs die selbigem zuerkannte Begründungsfunktion epistemologisch legitimiert, d.h. insbesondere ob der Gebrauch eines Grundbegriffes zu widersprüchlichen Konsequenzen führt. Damit handelt es sich im Verhältnis zwischen terminus a quo und Grundbegriff um ein epistemisches bzw. logisch-begriffliches Begründungsverhältnis, dem im Folgenden die Aufmerksamkest gilt.

32 Traditionell bezeichnen diese Termini „in Erinnerung an eine Kette von syllogistischen Schlüssen den Ausgangs- bzw. Endpunkt eines schlüssigen Argumentationsprozesses“ (Lorenz, K., „Terminus“, in: Mittelstraß, Jürgen (Hrsg.), Enzyklopädie Philosophie und Wissenschaftstheorie, Stuttgart und Weimar 1996, Band 4, S. 234-236, hier: S. 236), wobei im Folgenden nicht eine einzelne formallogische Schlusskette, sondern der innertheoretische Zusammenhang des terminus a quo mit einem Grundbegriff zentral steht. 


\section{Münchhausen}

Das Verhältnis zwischen einem terminus a quo und dessen Grundbegriff zeigt, inwiefern die theoretische Gültigkeit der Grundbegriffe für die explanatorischen Zwecke der Theorie legitimiert ist. Die theoretische Geltungsfrage geht demnach der eigentlichen Erklärung des Explanandums logisch vorher, d.h. letztere rekurriert auf die Voraussetzungsebene. Das Verhältnis des terminus a quo zu einem Grundbegriff ist demnach ein deduktives, weil der Grundbegriff hinsichtlich seines gültigen Gebrauches mittels des klassischen Prinzips der zureichenden Begründung (bzw. dem Satz vom zureichenden Grund) eingeholt werden kann. Dieser logisch-begrifflichen Analyse kommt hier nun insofern ein methodologischer Status im Sinne einer Prüfungsfunktion zu, als dass mittels der logisch-deduktiven Analyse die Legitimationsgründe von Grundbegriffen expliziert werden können, ohne dass damit allerdings eine entsprechende Forderung nach einer Letztbegründung einhergehen muss. Diesem innertheoretischen Begründungsverhältnis drohen dabei dieselben „problematischen Alternativen ${ }^{\text {“33 }}$ wie jedem deduktiven Begründungsansatz auch: ${ }^{34}$

(1) An erster Stelle steht dabei der unendliche Begründungsregress (regressus ad infinitum), „der durch die Notwendigkeit gegeben erscheint, in der Suche nach Gründen immer weiter zurückzugehen, der aber praktisch nicht durchführbar ist und daher keine sichere Grundlage liefert ${ }^{\text {" }}{ }^{35}$. Dies wäre der Fall, wenn sich der terminus a quo als unzureichend herausstellt. Der Grundbegriff würde damit seiner Geltung nach aufgehoben und stets nach weiteren termini a quo verlangen, d.h. seinerseits zu einem zu erklärenden terminus ad quem werden.

(2) Der einzige Ausweg aus einem solchen Regress ist der logische Zirkel, „der dadurch entsteht, daß man im Begründungsverfahren auf Aussagen zurückgreift, die vorher schon als begründungsbedürftig aufgetreten waren " ${ }^{136}$. Ein Begriff (oder Satz) wird so als seine eigene Begründungsbasis eingeführt. Nach dem obigen Schema hieße das, dass der Grundbegriff selbst oder ein aus diesem folgender Begriff, ein terminus ad quem, zugleich als terminus a quo fungiert.

(3) Dem Zirkel lässt sich dann am Ende nur noch mit dem dogmatischen Abbruch entkommen, „der zwar prinzipiell durchführbar erscheint, aber eine willkürliche Suspendierung des Prinzips der zureichenden Begründung involvieren würde ${ }^{\text {“37}}$, der also insofern keine Begründung mehr liefert, als dass gegenüber einer Begründungsbasis „die Verabredung

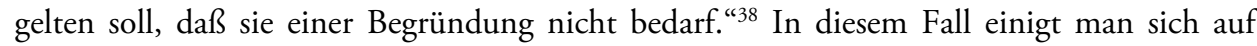
einen Grundbegriff und legt diesem ungeachtet seines begründungstheoretischen Defizits epistemische Geltung bei.

\footnotetext{
${ }^{33}$ Mittelstraß, Jürgen, „Münchhausen-Trilemma“, in: Mittelstraß 1996, Band 2, S. 945-946, hier: S. 945.

${ }^{34}$ Vgl. dazu Albert, H., Traktat über kritische Vernunft, Tübingen 1991, S. 13-18.

${ }^{35}$ Ebd., S. 15.

${ }^{36}$ Ebd.

${ }^{37}$ Ebd.

${ }^{38}$ Ebd.
} 


\section{SMT revisited: methodenkritische Analyse der Grundbegriffe}

Die in Abschnitt 1 aufgestellten Grundbegriffe werden im Folgenden der methodenkritischen Analyse unterzogen. Bewusstsein wird gemäß der These des repräsentativen Realmonismus bzw. SMT als metamentale Repräsentation konzipiert. Diese übergeordnete repräsentationale Struktur des Bewusstseins spezifiziert sich ihrerseits in zwei weitere Bestimmungsverhältnisse: Mit Blick auf den Weltbezugs bürgt der erkenntnistheoretische (repräsentative) Realismus für die epistemische Gegebenheit der Welt sowie deren Zugänglichkeit, d.h. der Realismus übt hier eine wirklichkeitskonstituierende Funktion aus. Der Teleofunktionalismus wiederum schreibt dem System in praktischer Hinsicht inhärente innerweltliche Interessen (Fortpflanzung und Arterhaltung) zu und ermöglicht somit dessen Handlungsfähigkeit in der empirischen Welt.

Daraus ergibt sich auf Basis des methodenkritischen Analyseschemas folgende Übersicht:

\begin{tabular}{|c|c|c|c|}
\hline $\begin{array}{c}\text { Voraussetzungsebene } \\
\text { (terminus a quo) }\end{array}$ & Repräsentationalismus & Realismus & Teleofunktionalismus \\
\hline $\begin{array}{c}\text { Theorieebene: SMT - } \\
\text { Grundbegriffe } \\
\text { (repräsentativer } \\
\text { Realmonismus) }\end{array}$ & Repräsentation & $\begin{array}{c}\text { realistische } \\
\text { Gegebenheit und } \\
\text { Zugänglichkeit } \\
\text { der Welt }\end{array}$ & $\begin{array}{c}\text { Vevolutive } \\
\text { Verhaltenssteuerung }\end{array}$ \\
\hline $\begin{array}{c}\text { Erklärungsebene } \\
\text { (terminus ad quem) }\end{array}$ & Zum system und Zur Welt & $\begin{array}{c}\text { Intentionale Beziehung } \\
\text { rentationale }\end{array}$ & Handlungssteuerung \\
\cline { 2 - 4 } & \multicolumn{2}{|c|}{ phänomenales Selbst } \\
\hline
\end{tabular}

(Abb. 2)

\section{Repräsentationalismus}

Zur Erklärung der modelltheoretischen Architektur übt der Grundbegriff der (metamentalen) Repräsentation die Funktion aus, die Beziehung zwischen sinnlich aufgefasstem Gegenstand und dessen mentalem Gegebensein sowie die metarepräsentationale Erfassung zu ermöglichen. Sowohl die repräsentative Erfassung der Wirklichkeit bzw. die für das System zugänglichen Eigenschaften (Realitätsmodell) als auch die metamentale Selbstmodellbildung beruhen auf der repräsentationalen Gesamtarchitektur des Gehirns, wobei die spezifische (analoge) Repräsentationsbeziehung hier das entscheidende Glied zwischen System und Welt bildet. Gemäß des naturalistischen Begründungsdenkens beruht der SMT zugrundeliegende Repräsentationalismus auf dieser Repräsentationsbeziehung. Damit geht die theoretische Festlegung einher, dass dieser erkenntnistheoretische Zusammenhang, das repräsentationale Selbst- und Weltverhältnis des Systems, konstitutiv für das Bewusstsein ist. Der naturalistische Grundbegriff der (metamentalen) Repräsentation führt zwecks der Geltungsbegründung als terminus a quo die Repräsentationsbeziehung an. Folgendermaßen gestaltet sich daher der geltungstheoretische Diskurs in SMT (Abb. 3): 


\author{
terminus a quo: \\ Repräsentationsbeziehung \\ $\downarrow$ \\ Grundbegriff:
}

(metamentale) Repräsentation

(Abb. 3)

Die Begründung theoretischer Geltung obliegt nach dem methodenkritischen Analyseschema dem terminus a quo. Insofern gilt es jetzt, das Verhältnis zwischen diesem und dem Grundbegriff zu bestimmen: Stellt der terminus a quo eine zureichende Begründung für den Grundbegriff dar? Wie begegnet SMT der trilemmatischen Begründungsstruktur, also den begründungslogischen Gefahren des Regresses, des Zirkels und schließlich des Abbruches?

Ein infiniter bzw. repräsentationaler Regress würde dann vorliegen, wenn zur Begründung der Repräsentation, also des epistemischen Verhältnisses von System und Welt eine weitere repräsentationale Ebene veranschlagt würde, wie zum Beispiel Sinnesdaten. Dieser Rekurs auf immer wieder vorgelagerte Repräsentationen wird nun durch den angegebenen terminus a quo vermieden, indem ebendiese Beziehung der gesamten selbstmodelltheoretischen Erklärung des Bewusstseins vorausgesetzt wird. ${ }^{39}$ Damit ist zunächst dafür gesorgt, dass in der Erklärung des phänomenalen Selbst kein repräsentationaler Regress droht. Wie sieht es nun aber mit dem Zirkel aus, den Albert bereits als einzig möglichen Ausweg aus dem Regress aufgezeigt hat?

Der terminus a quo müsste eigentlich erklären, wie die (metamentale) Repräsentation möglich ist. Stattdessen aber führt der Repräsentationalismus als terminus a quo lediglich eine Analyse seines Grundbegriffes mit sich, womit in geltungstheoretischer Hinsicht nichts gewonnen wird, weil der zu erklärende Begriff, also die Repräsentationsbeziehung, im terminus a quo lediglich wiederholt wird. Zur Erklärung dieses Verhältnisses wird gerade diese Repräsentationsbeziehung selbst vorausgesetzt: Es wird vorausgesetzt, dass das System die Welt abbilden kann. SMT beschreibt dann lediglich, wie unter dieser Voraussetzung, nämlich durch metarepräsentationale Verarbeitung, phänomenales Bewusstsein entsteht. Damit wird aber gerade auf einen Begründungszusammenhang rekurriert (terminus a quo), der seinerseits eigentlich erklärt werden soll, nämlich wie ein solch gehaltvolles repräsentationales Verhältnis zwischen System und Welt allererst möglich ist. Um dem Begründungsregress zu entgehen, geht SMT also vom empirischen Sachverhalt der Repräsentation aus, während die geltungstheoretische Frage, wie Repräsentation selbst möglich ist, nicht mehr beantwortet wird, weil die modelltheoretische Erklärung der repräsentationalen System-Welt-Beziehung immer schon unter Voraussetzung derselben ausgeführt wird. ${ }^{40}$

\footnotetext{
${ }^{39}$ Esken und Heckmann verhandeln diese Problemstellung als die Diskussion um ursprüngliche und metamentale Repräsentation (vgl. Abschnitt 1) und verbleiben damit innerhalb des repräsentationalistischen Gesamtrahmens, während mittels der grundbegrifflichen und methodenkritischen Analyse dieser Theorierahmen selbst thematisiert werden kann.

${ }^{40}$ Der gleiche Punkt lässt sich auch als petitio principii explizieren: Erklärt werden soll die psychologische Instantiierung von Bewusstseinserlebnissen (insbesondere der phänomenalen Subjektivität). Allerdings sind die
} 
Als erstes methodenkritisches Resultat lässt sich also festhalten, dass die Begründung bzw. genauer die geltungstheoretische Absicherung des Grundbegriffes der Repräsentation dem Zirkel anheimfällt. Der Begründungsdiskurs findet im Repräsentationalismus ein jähes Ende, indem dieser die Repräsentationsbeziehung faktisch, d.h. nicht weiter begründet, als gegeben und gültig festschreibt, was letztlich einem dogmatischen Abbruch gleichkommt.

\section{Realismus}

Die realistische Gegebenheit der Welt, die den Repräsentationen ihre objektive Basis verleiht, beruht auf dem realistischen Credo, dass „Existenz und Beschaffenheit der Welt unabhängig vom menschlichen Geist gegeben sind“ ${ }^{411}$ sowie mittels wissenschaftlicher Methoden zugänglich sind. Dementsprechend fällt das methoden-kritische Analyseschema aus:

\section{terminus a quo:}

natürliche und epistemisch zugängliche Wirklichkeit

$$
\text { Grundbegriff: }
$$

realistische Gegebenheit und Zugänglichkeit der Welt

(Abb. 5)

Wie bei jedem Grundbegriff stellt sich auch im Falle des Realismus zuerst die Frage, wie der infinite Regress vermieden kann. Dieser sähe so aus, dass zur Begründung der realistisch gegebenen phänomenalen Realität bzw. der empirischen Natur eine Realität höherer Ordnung vorausgesetzt werden müsste, die ihrerseits wiederum auf eine Realität noch höherer Ordnung rekurrieren würde, bis eine „letzte“ Realität, ausgewiesen wird. Dieses Problem wird gelöst, indem die natürliche (physikalisch-biologische) Wirklichkeit als eine solche letzte bzw. Realität höchster Ordnung, d.h. als objektive Realität gesetzt wird. Ein Blick auf den terminus a quo offenbart hier dann aber auch einen Zirkel: Die im Grundbegriff veranschlagte Welt ist auf der Voraussetzungsebene ihrer selbst vorausgesetzt, d.h. zur Begründung des objektiven Status der natürlichen Wirklichkeit dient nur der faktische (empirische) Rekurs auf dieselbe. Das ist jedoch geltungstheoretisch ungenügend, weil ein

\footnotetext{
Elemente der Erklärung insgesamt auch nur wieder Repräsentation. Damit entsteht die Frage, wie aus der Repräsentation heraus gehaltvolle Repräsentation begründet (und nicht nur naturwissenschaftlich erklärt) werden kann. Zur Begründung der Repräsentation dienen wiederum nur Repräsentationen, was auf eine petitio hinausläuft, da der Beweisgrund für die Repräsentation, also deren realistischer und damit Gehalt stiftender Bezug auf ein Gegebenes, ungerechtfertigterweise in eine Repräsentation (die des Gehirns) gelegt wird.

Oder aber als naturalistische Subreption: Erschlichen wird hier nämlich, dass reine Physis, die per definitionem nicht bewusst ist, Bewusstsein hervorbringt. Diese grundlegende methodische Schwierigkeit natura-listischer Bewusstseinstheorie hat auch schon Walshe treffend analysiert; vgl. dazu Walshe, F.M.R.: „The Neurophysiological Approach to the Problem of Consciousness“, in: Critchley, M. - O'Leary, J. - Jennett, B. (Hrsg.), Scientific Foundations of Neurology, London 1972, S. 181-189.

${ }^{41}$ Abel, G., „Realismus, III. Analytische Philosophie“, in: Historisches Wörterbuch der Philosophie, Band 8, Sp. 162-169, hier: Sp. 163.
} 
solcher faktischer Rekurs keine zureichende Begründung liefert, sondern formal gesehen nur eine analytische Wiederholung des im Grundbegriff konstatierten Sachverhalts darstellt. Hier führt der Realismus mit der epistemisch nicht mehr ausgewiesenen Festschreibung der natürlichen Welt als Welt höchster Ordnung sowohl in ontologischer als auch in epistemologischer Hinsicht realmonistisch in den dogmatischen Abbruch.

\title{
Teleofunktionalismus
}

Der Teleofunktionalismus bezieht seine objektive Geltung aus der Evolutionsbiologie, der zufolge der Sinn und Zweck aller Organismen ihre biologische Selbst- bzw. Arterhaltung ist.

\author{
terminus a quo: \\ Interesse einer jeden Art an ihrer biologischen Erhaltung \\ $\downarrow$ \\ Grundbegriff: \\ evolutive Verhaltenssteuerung \\ (Teleofunktionalismus)
}

(Abb. 6)

Hier steht der Teleofunktionalismus hinsichtlich seines teleologischen Moments zentral. ${ }^{42}$ Die Funktion des teleologischen Moments liegt in der Angabe eines normativen Kriteriums zur handlungsermöglichenden Selektion von Repräsentaten, d.h. der Auswahl derjenigen Repräsentate, die dem System zur Arterhaltung relevante kausale Zusammenhänge der Welt zugänglich machen. Damit wird dem Organismus letztlich Handlungsfähigkeit im Sinne eines auf dessen Erhaltung hin ausgerichteten Wirkens in der empirischen Wirklichkeit ermöglicht, so dass der Organismus ein ethischer Agent wird. Der terminus a quo hätte unter anderem anzugeben, wieso das spezifische Interesse an biologischer Erhaltung den Zweck des Handelns bzw. das genannte teleologische Zusatzkriterium angibt. Die Begründung dieser Auswahl des Kriteriums entscheidet der Evolutionismus jedoch unter Verweis auf das biologische Leben und auf entsprechende biologische Theorien wie beispielsweise diejenige Darwins. Somit rekurriert der teleofunktionalistische Grundbegriff auf die empirischen handlungsfähigen Organismen, deren auf dem Zweck der Arterhaltung basierende Handlungsfähigkeit eigentlich erklärt werden sollte, als Bestimmung des terminus a quo. Da dieser aber nichts weiter enthält als eine Analyse seines Grundbegriffs, zeigt sich auch im Falle des Evolutionismus ein unzureichender, weil zirkulärer terminus a quo. Die letztlich programmatische Festschreibung des evolutiven Zusatzkriteriums führt den Teleofunktionalismus in geltungstheoretischer Perspektive in den dogmatischen Abbruch.

\footnotetext{
${ }^{42}$ Die funktionale Realisierung von Bewusstsein kann hier ausgeklammert werden, insofern für die weitere Analyse das normative Moment der Zwecksetzung entscheidend ist.
} 


\section{Beweis der Gegenthese: Bewusstseinstheorie ohne Wissenstheorie ist ungültig}

Mit den aufgezeigten geltungstheoretischen Inkonzinnitäten ist der erste Schritt der reductio ad absurdum der realmonistischen These abgeschlossen. Die trilemmatischen Grundbegriffe implizieren ihrerseits widersprüchlichen Aussagen, die SMT und damit die These ad absurdum führen.

\section{Gehirnparadox}

Das Gehirnparadox beruht auf einem repräsentationalen Dilemma. Einerseits tritt das Gehirn (bzw. der Organismus) als der Produzent von Repräsentationen auf. Andererseits verlangt aber der Realitäts-Modellcharakter unseres Bewusstseins, dass wir in diesem unseren Bewusstsein immer nur mit Modellen bzw. Selbstmodellen operieren ${ }^{43}$ und so die vorausgesetzte objektive Wirklichkeit immer nur als modellierte Wirklichkeit im Bewusstsein haben. Auf den Widerspruch zwischen der Eigenschaft des Gehirns als Produzent von Repräsentaten und andererseits dessen ausschließlicher Zugänglichkeit mittels Repräsentationen wird in SMT nicht eigens reflektiert. Der Sache nach müsste man aus erkenntnistheoretischer Perspektive das neurowissenschaftlich erforschte Gehirn zunächst auch als Repräsentat thematisieren (was nicht ausschließt, dass es selbst auch Repräsentate, aber dann einer anderen Ordnung, hervorbringt). Seine Veranschlagung als der ursprüngliche Produzent von Repräsentation überhaupt und damit das naturalistisch postulierte Kausalverhältnis zwischen Welt und Bewusstsein kann aber letztlich nicht aufrecht erhalten werden, da der empirische Rahmen zur Erklärung von Bewusstsein (die natürliche Wirklichkeit) zugleich das konstituierte Bewusstseins bildet, in dem die Erklärung stattfindet. SMT zufolge müsste das Gehirn Ursache der Repräsentation und gleichzeitig Resultat der Repräsentation sein. Aus diesem ungelösten Widerspruch resultiert in geltungstheoretischer Hinsicht dahingehend eine Einschränkung des naturalistischen Erklärungsanspruches, dass die empirisch grundgelegte Wirklichkeit nicht in einem ausschließlichen Kausalverhältnis zum Bewusstsein stehen kann, da beide epistemisch gesehen immer gleichursprünglich gegeben sind.

\section{Zweckaporie}

Neben diesem repräsentationalen Dilemma resultieren auch aus dem Postulat biologischer Zwecksetzung dieselben strukturellen Schwierigkeiten, die mit dem Teleofunktionalismus als entfinalisierter Teleologie zusammenhängen und in eine begründungstheoretische Aporie führen. Während für die Handlungsfähigkeit ein normatives Selektionskriterium gefunden werden muss, dürfen im umfassenden naturalistischen Kontext nur nomologische Handlungen vorliegen, also solche, die vollständig im kausalen Gefüge der Welt bestimmt sind. So muss ein Handlungszweck formuliert werden, der aber eigentlich keiner sein darf, wenn die

\footnotetext{
${ }^{43}$ Man denke an den programmatischen Ausspruch Metzingers: „Werden wir nicht errechnet, so gibt es uns nicht." (Metzinger 1999, S. 284).
} 
kognitiven Systeme diesen mit Notwendigkeit ausführen sollen. Wie schon die konstitutive Funktion des Gehirns widersprüchlich ist, scheitert auch die Begründung der praktischen Handlungsfähigkeit eines repräsentational fundierten Bewusstseins, weil dieses ohne einen Zweckbegriff bzw. ein normatives Kriterium nicht handlungsfähig wäre, die aber im naturalistischen Kontext kategorisch ausgeschlossen werden. Streng genommen dürfte das Problem, kausal relevante von kausal irrelevanten Zusammenhängen in der Wirklichkeit unterscheiden zu müssen, im naturalistischen Kontext gar nicht aufkommen, wenn man den Anspruch der Nomologizität ernst nehmen würde. Statt dessen schleicht sich aber die Notwendigkeit einer Zweck- bzw. Normsetzung ein, da ein entsprechendes Kriterium, das die genannte Selektion ermöglicht, explanatorisch notwendig ist. Der Naturalist kommt also um die Setzung eines normativen Kriteriums nicht umhin, das es seiner Theorie zufolge eigentlich gar nicht geben dürfte.

Die These des repräsentativen Realmonismus wird durch die grundbegrifflichen Aporien bzw. das Münchhausen-Trilemma und die daraus resultierenden Widersprüche ad absurdum geführt. Der postulierte epistemologische Primat der Natur muss damit zugunsten der Gegenthese aufgegeben werden: Das Bewusstsein ist nicht monokausales bzw. ausschließliches Produkt der Natur und damit auch irreduzibel selbstbezüglich sofern es in jedem Wirklidnkeits bezug epistemisch voransgesehzt werden muß. Bewusstseinstheorie verlangt daher notwendig nach einer epistemologischen Reflexion auf die Voraussetzungsebene, d.h. auf die Legitimationsbasis der Grundbegriffe. ${ }^{44}$

\section{Das geltungstheoretische Problembewusstsein und die Selbstbezüglichkeit des Bewusstseins}

„aber dieses Bild hält sich durchaus nicht für ein Bild. [...] also [...], daß es nicht gebildet sein will.“ ${ }^{45}$

Die hier aufgewiesene irreduzible epistemische Selbstbezüglichkeit ist strukturell bedingt durch die Gleichursprünglichkeit von Form und Inhalt: Der Inhalt bzw. die Welt (also das, was das Subjekt im Bewusstsein vollzieht) ist gleichursprünglich mit der Form als der subjektiven (theoretischen wie praktischen) Gegebenheit dieser Welt gegeben. Zufolge der Form-Inhalt-Beziehung des Bewusstseins, d. h. ein real Gegebenes (Inhalt), zu dem das Subjekt in theoretischen Gegebenheits- und praktischen Handlungsweisen (Form) im Verhältnis steht, rekurrieren wir auf die objektive Wirklichkeit bzw. auf die naturwissenschaftlich und naturalistisch veranschlagte Natur notwendig in Form spezifischer phänomenaler,

${ }^{44}$ Damit soll nicht der gesamte Erklärungsanspruch naturalistischer Theorien bestritten werden. Ohne Zweifel ist es für eine gründliche Erforschung des Bewusstseins unumgänglich, auch die materialen Konstitutionsbedingungen einzubeziehen. Wie dies im Detail möglich ist, muss methodisch die Wissenschaftstheorie der Neurowissenschaften klären, d.h. deutlich machen, wie die neurowissenschaftlichen Erkenntnisse zu bewerten sind. Das kategorische Urteil der epistemischen Ungültigkeit verdankt sich hier zunächst dem Beweisgang der reductio ad absurdum und dient hier vornehmlich zur Kenntlichmachung der Notwendigkeit einer wissenstheoretischen Reflexion, die im naturalistischen Kontext als generell unmöglich veranschlagt wird, d.h. jegliche Geltungsfragen werden in den umfassenden naturalistischen Kontext eingeordnet, ohne diesen selbst zu thematisieren.

${ }^{45}$ Fichte, J.G., Ueber das Verhältniß der Logik zur Philosophie oder Transscendentale Logik (Oktober bis Dezember 1812), Hamburg 1982, Blatt 34v. 
kognitiver sowie voluntativer Gegebenheitsweisen. Wenn somit also als Ursache für diese Gegebenheitsweisen bzw. das Bewusstsein ausschließlich etwas angegeben werden kann, das nur mit diesem Bewusstsein selbst überhaupt als eine objektive Wirklichkeit gegeben ist, dann kann die biologische Wirklichkeit nicht mehr als letzte Ursache fungieren. Konkret heißt das: Da die empirische Wirklichkeit des Naturalisten bzw. die Natur uns Menschen auch nur durch das Bewusstsein gegeben ist, kann damit auch die Natur nur gemäß der Form des Bewusstseins repräsentiert werden und hängt somit ihrerseits auch von den Konstitu-tionsbedingungen des Bewusstseins ab.

Das geltungstheoretische Versäumnis der naturalistischen Erklärung von Bewusstsein liegt also darin, den für eine ursächliche Erklärung des Bewusstseins epistemisch ungenügenden Status des grundlegenden Repräsentationsverhältnisses inklusive des Realismus und Teleofunktionalismus bzw. die selbstbezügliche Form-Inhalt-Beziehung, der zufolge der phänomenale Wirklichkeitsbezug als ebenso konstitutiv wie die natürlichen Grundlagen zu veranschlagen ist, nicht zu berücksichtigen. Oder um das Anfangszitat dieses Abschnitts aufzugreifen: Hier geriert sich ein repräsentationaler Inhalt (in der Terminologie Fichtes ein Bild), seinen epistemischen Status zu überbeanspruchen und unabhängig von seiner repräsentationalen Form ein objektiv Wirkliches sein zu wollen, d.h. seine repräsentationale Gegebenheit geradeweg für eine objektive bzw. reale Gegebenheit hält. Es ist dabei nicht nötig, den offensichtlichen Anteil der biologischen Lebensgrundlage zu leugnen und in einen intelligiblen Monismus oder einseitigen Idealismus bzw. Subjektivismus zu verfallen. Die gesamte Problematik der Theoretisierung des Bewusstseins als einer selbstbezüglichen Struktur muss dementgegen zunächst auf ein methodisch vorgängiges Niveau verlegt werden, d.h. es darf nicht von einzelnen Konstitutionsmomenten, wie dem Gehirn, der Natur oder einem geistigen Bezug ausgegangen werden, sondern es muss gezeigt werden, welchen Momenten welche konstitutive Funktion zukommt. Nach der kritischen Revision der naturalistischen Grundbegriffe stellt sich also die Notwendigkeit, die spezifische FormInhalt-Beziehung des Bewusstseins mit Blick auf die Geltung der Grundbegriffe methodisch zu verautworten.

\section{Wissenstheorie: transzendentaler Dualismus}

Die drei aus naturalistischer Sicht notwendigen Grundbegriffe der Repräsentationsbeziehung, der realen Gegebenheit der Welt und der evolutiven Verhaltenssteuerung fungieren nur auf Kosten einer theoretisch ungültigen Argumentation als exklusive konstitutive Kategorien. Die begriffslogische Analyse hat gezeigt, dass im Falle der Theoretisierung des Bewusstseins die zu erklärenden Bewusstseinsleistungen nur unter zeitgleicher Inanspruchnahme derselben erschließbar sind. Das Bewusstsein ist also insofern selbstbezüglich, als dass sämtliche Realität durch die Form desselben vermittelt ist, während wir mit Blick auf unsere natürliche Gegebenheit auch unserer materiellen Grundlagen sicher sein können. Die realmonistischen Grundbegriffe brauchen daher auch nicht in toto aufgegeben zu werden. Vielmehr muss der epistemische Status der biologischen Gegebenheit reflektiert und zu weiteren konstitutiven Momenten in Beziehung gesetzt werden. Nur zu oft werden die einzelnen Positionen begrifflicher Antagonisten wie Körper und Geist, Leib und Seele, 
Begriff und Anschauung absolut gesetzt und eine entsprechende Reduktion häufig Zugunsten einer materialistischen Bestimmung gefordert.

Der wissenstheoretische Anteil in der Formulierung der These zielt auf die methodische Kontrolle dieser begriffslogischen Beziehungen mittels einer transzendentalen Reflexion bzw. einer reflexiven Epistemologie mit einem eigenem, d.h. hier insbesondere nichtempirischem, Geltungsanspruch, der durch die reductio der These erwiesen ist. Er gründet selbst auf der strukturellen Form-Inhalt-Beziehung: Die Position des transzendentalen Dualismus besagt daher, dass neben der explanatorischen Reflexion auf den Untersuchungsgegenstand bzw. den Inhalt immer auch eine epistemologische Reflexion auf die Form der Untersuchung notwendig ist, der zufolge die Grundbegriffe geltunstheoretisch geprüft werden. Insofern formuliert die Gegenthese eine methodisch basierte Erwiderung auf die These, die zunächst nur explanatorische Aussagen über den Untersuchungsgegenstand machte. Diese methodische Transformation trägt der spezifischen selbstbezüglichen Struktur des Bewusstseins Rechnung, die sich nur berücksichtigen lässt, wenn man den epistemischen Bestimmungen des Bewusstseins prinzipiell ein eigenständiges Geltungsmoment zuerkennt und damit unter anderem auch die Gegebenheitsweise der als objektiv veranschlagten Realität epistemisch bestimmen kann. Die methodische Kontrolle besteht darin, die konstitutiven Geltungsmomente einer Bewusstseinstheorie, wie sie in den Grundbegriffen festgelegt werden, zu unterscheiden, um aus ihnen dann die Theorie bzw. die Erklärung des Bewusstseins zu formulieren. Es obliegt einer solchen reflexiven Bewusstseinstheorie letztlich die Aufgabe, den genaueren Zusammenhang der verschiedenen Geltungssphären, hier anhand der natürlichen und der intelligiblen gezeigt, im Detail zu bestimmen. Diese Aufgabe beinhaltet - ex negativo - die Vermeidung der repräsentationalistischen und teleofunktionalistischen Aporien. Die kritische Überprüfung realistischer Existenzunterstellungen, die Explikation aller Geltungsmomente sowie die explanatorische Instrumentalisierung der daraus folgenden Grundbegriffe werden unter Berücksichtigung der Form-Inhalt-Beziehung bzw. der irreduziblen Selbstbezüglichkeit in einer idealrealistischen Dialektik ausgeführt, die das reale Gegebenheitsmoment mit dem idealen Bezugsmoment, das als ebenso konstitutiv aufgewiesen wurde, vermittelt. ${ }^{46}$

${ }^{46}$ Vgl. dazu vom Verfasser „Grundlagen und Voraussetzungen der Leib-Seele-/Körper-Geist-Dichotomie in der gegenwärtigen Philosophie des Geistes“, in: Asmuth, C. (Hrsg.), Transzendentalphilosophie und Person. Leiblichkeit - Interpersonalität - Anerkennung, Bielefeld 2007, S. 23-40 und „Fichtes transzendentalphilosophische Methode und die Leib-Seele-/Körper-Geist-Dichotomie“, in: ebd., S. 90-106. 